Mini Review

\title{
Ocular myasthenia: clinical features and diagnosis
}

\begin{abstract}
Myasthenia affecting the eyes continues to be a challenging diagnosis to make. Confirmatory tests may fail to positively identify the clinical suspicion. Approximately one third of the patients have a history of unilateral/bilateral fatiguing ptosis. Positive acetylcholine receptor antibodies are found in half of the patients diagnosed with ocular myasthenia and in $75 \%$ of those with the generalized form. Clinical signs and symptoms are of prognostic value in establishing the diagnosis. A multidisciplinary team approach is required.
\end{abstract}

Keywords: myasthenia gravis, acetylcholine receptor antibodies, variable diplopia

\author{
Volume 7 Issue 4 - 2017
}

\author{
Evangelos Drimtzias, Janice Hoole, lan \\ Simmons \\ Department of Ophthalmology, Leeds Teaching Hospitals, St. \\ James University Hospital, United Kingdom
}

Correspondence: Evangelos Drimtzias, Department of Ophthalmology, Leeds Teaching Hospitals NHS Trust, St. James University Hospital, Beckett Street, Leeds, West Yorkshire, LS9 7TF, United Kingdom, Tel +44(0) I | 32433 | 44, Fax +44(0)I I32 I66055, Email evangelos.drimtzias@nhs.net

Received: April 06, 2017 | Published: September 08, 2017

\section{Introduction}

Myasthenia Gravis (MG) is a chronic acquired autoimmune disorder of the neuromuscular junction which produces fatigue of the skeletal muscles. Although weakness may affect any muscle, MG has a distinct predilection for involvement of the extraocular muscles (EOM). MG patients are therefore categorized into two groups, the ocular MG (OMG) and the generalized group. The diagnosis of OMG is challenging and not always clinically evident since symptoms and signs can mimic other conditions.

The age distribution is bimodal with incidence peaks in the 20 s for women and in the 40 s for men. ${ }^{1} \mathrm{MG}$ is relatively uncommon in the paediatric population with children accounting for approximately 10 $15 \%$ of the cases annually. ${ }^{2}$

\section{Ocular manifestations}

The initial presentation is limited to pure ocular symptoms. More than three quarters of MG patients present with visual complaints of eyelid ptosis and diplopia, however, all patients develop ocular symptoms at some stage in the course of generalised disease. ${ }^{3}$ Approximately, $90 \%$ of children with MG will have ophthalmic features such as eyelid ptosis or ophthalmoplegia. ${ }^{4}$

Eyelid ptosis and ophthalmoparesis are the most common manifestation of OMG. Ptosis may be unilateral or bilateral and it is usually asymmetric. Variation in severity is the hallmark of MG. MG is the only diagnosis to consider with a history of alternating or recurrent painless ptosis. Nearly $90 \%$ of patients who present with diplopia have associated eyelid ptosis and this combination should immediately bring the diagnosis of MG to forefront. ${ }^{3}$ Orbicularis oculi weakness in combination with ptosis or ophthalmoparesis is a strong indicator of $\mathrm{MG}^{5}$

Approximately, half of MG patients who present with ocular manifestation develop generalized disease within 6 months and up to $80 \%$ will generalize within 2 years. ${ }^{6}$ In contrast, OMG presenting in children is less likely to progress to generalization. Previous studies have reported rates of generalization between $7-36 \%$ in the paediatric population. ${ }^{7}$ However, in the study conducted by Nagia et al, a much lower overall conversion rate of $21 \%$ was reported. ${ }^{8}$

According to previous authors, it is likely that patients who remain with symptoms localized to the EOM for more than two years will not generalize. ${ }^{9}$ However, these patients should not be assured that the risk of conversion after 2 years is minimal, since previous reports showed that $30 \%$ of patients convert beyond two years and therefore should continue to be monitored in the long-term. ${ }^{8}$

\section{Factors affecting conversion rates}

Several factors have been postulated to affect conversion rates. Previous reports showed a trend toward frequent progression to generalized form of MG in older individuals. ${ }^{10}$ In addition, high AChR antibody titers at presentation have been found to be associated with increased risk of generalization. ${ }^{11}$ Previous studies showed increased risk of conversion with thymoma, ${ }^{12}$ while there is some evidence in the literature showing thymectomy to be effective in controlling juvenile OMG by reducing the risk of generalization. ${ }^{13}$ Retrospective studies have suggested reduced rates of progression in patients treated with steroids. ${ }^{14}$ However, whether early steroid treatment may prevent generalization is debated since there is no conclusive evidence from prospective studies.

\section{Differential diagnosis}

OMG may mimic any pupil-sparing ocular motility disorder, including third, fourth and sixth cranial nerve palsies, central gaze disorders such as internuclear ophthalmoplegia and one and a half syndrome, as well as chronic progressive external ophthalmoplegia and thyroid eye disease. The diagnosis of OMG may often be made based on clinical grounds since no other conditions are consistent with patient's presenting manifestations, but most clinicians and patients feel more confident with therapeutic decisions in light of a positive confirmatory test. However, even these tests may fail to positively identify the clinical suspicion.

\section{Diagnostic testing}

The Edrophonium test provides confirmatory evidence of MG, however, it lacks specificity and it may be complicated by bradycardia or bronchiolar constriction. The ice pack, sleep and rest tests are other clinical tests that may be substitute for the edrophonium evaluation. These tests are simple and can be done quickly in the office without serious complications. They may be performed in patients in whom pharmacologic testing is contraindicated.

Repetitive nerve stimulation (RNS) has low positivity in both OMG and GMG groups. Positivity was reported at $51.78 \%$ and in $82.35 \%$ of the cases respectively. ${ }^{15}$ Single fiber electromyography (SFEMG) is sensitive and specific for OMG but is technically challenging and not widely available. ${ }^{16}$ 
Clinicians may order 3 types of AChR antibody test: binding, blocking or modulating. The binding antibody is highly specific but is thought to be less sensitive in OMG compared to generalized disease ${ }^{17}$ However, in the study conducted by Peeler et al, a higher sensitivity of AChR antibody was demonstrated in OMG patients in the rate of $70.9 \%{ }^{18}$ The authors suggested that older age, male sex and progression to generalized disease were significantly associated with a positive antibody result.

Antibodies against muscle specific kinase (MuSK) are detected in around $5 \%$ of patients with GMG. In addition, it can be found positive in about a third to a half of patients with generalized disease but with negative AChR antibody. ${ }^{19}$ In around $15 \%$ of subjects with GMG and in up to $50 \%$ of patients with the ocular form of the disease, AChR and MuSK antibody are reported as negative. These cases are classified as double seronegative group (dSNMG) ${ }^{20}$

\section{Conclusion}

Although MG is often considered the best understood autoimmune disorder, it remains a challenging disorder for the clinician. The diagnosis of OMG is not always clinically evident, as the pattern of deficits can mimic other conditions. Means of diagnosis are generally straightforward, as long as the condition comes to the clinician's mind. Clinical signs and symptoms are of prognostic and diagnostic value. Multitude of tests are important for the diagnosis of MG. Such tests must be used with a thorough appreciation of their limitations, however, so as not to inappropriately question a strong clinical diagnosis. ${ }^{21}$ However, a multidisciplinary team approach, including neurology and ophthalmic assessement, is required to establish the diagnosis.

\section{Acknowledgments}

None.

\section{Conflicts of interest}

None.

\section{Funding}

None.

\section{References}

1. Mantegazza R1, Baggi F, Antozzi C, et al. Myasthenia gravis (MG): epidemiological data and prognostic factors. Ann $N$ Y Acad Sci. 2003;998:413-423.

2. Phillips LH, Torner JC, Anderson MS, et al. The epidemiology of myasthenia gravis in central and western Virginia. Neurology. 1992;42(10):1888-1893.

3. Barton JJ, Fouladvand M. Ocular aspects of myasthenia gravis. Semin Neurol. 2000;20(1):7-20.

4. Andrews PI. Autoimmune myasthenia gravis in childhood. Semin Neurol. 2004;24(1):101-110.
5. Fouladvard M, Ledoux D, Barton JS. Ocular myasthenia gravis. Comprehensive Ophthalmology Update. 2005;6:25-36.

6. Kupersmith MJ, Latkany R, Homel P. Development of generalized disease at 2 years in patients with ocular myasthenia gravis. Arch Neurol. 2003;60(2):243-248.

7. Ortiz S, Borchert M. Long-term outcomes of pediatric ocular myasthenia gravis. Ophthalmology. 2008;115(7):1245-1248.

8. Nagia L, Lemos J, Abusamra K, et al. Prognosis of Ocular Myasthenia Gravis: Retrospective Multicenter Analysis. Ophthalmology. 2015;122(7):1517-1521.

9. Oosterhuis HJ. The natural course of myasthenia gravis: a long term follow up study. JNeurol Neurosurg Psychiatry. 1989;52(10):1121-1127.

10. Weizer JS, Lee AG, Coats DK. Myasthenia gravis with ocular involvement in older patients. Can J Ophthalmol. 2001;36(1):26-33.

11. Peeler CE, De Lott LB, Nagia L, et al. Clinical Utility of Acetylcholine Receptor Antibody Testing in Ocular Myasthenia Gravis. JAMA Neurol. 2015;72(10):1170-1174.

12. Hong YH, Kwon SB, Kim BJ, et al. Prognosis of ocular myasthenia in Korea: a retrospective multicenter analysis of 202 patients. J Neurol Sci. 2008;273(1-2):10-14.

13. Pineles SL, Avery RA, Moss HE, et al. Visual and systemic outcomes in pediatric ocular myasthenia gravis. Am J Ophthalmol. 2010;150(4):453-459.

14. Kupersmith MJ. Does early immunotherapy reduce the conversion of ocular myasthenia gravis to generalized myasthenia gravis? $J$ Neuroophthalmol. 2003;23(4):249-250.

15. Patil SA, Bokoliya SC, Nagappa M, et al. Diagnosis of myasthenia gravis: Comparison of anti-nicotinic acetyl choline receptor antibodies, repetitive nerve stimulation and Neostigmine tests at a tertiary neuro care centre in India, a ten year study. J Neuroimmunol. 2016;292:81-84.

16. de Entrambasaguas M, López-Bernabé R, López-Alemany M. Ocular myasthenia gravis: diagnostic aspects and evolution. Rev Neurol. 2007;44(7):397-403.

17. Grob D, Brunner N, Namba T, et al. Lifetime course of myasthenia gravis. Muscle Nerve. 2008; 37(2):141-149.

18. Peeler CE, De Lott LB, Nagia L, et al. Clinical Utility of Acetylcholine Receptor Antibody Testing in Ocular Myasthenia Gravis. JAMA Neurol. 2015;72(10):1170-1174.

19. Zhou L, McConville J, Chaudhry V, et al. Clinical comparison of muscle-specific tyrosine kinase (MuSK) antibody-positive and -negative myasthenic patients. Muscle Nerve. 2004;30(1):55-60.

20. Cortés-Vicente E, Gallardo E, Martínez MÁ, et al. Clinical Characteristics of Patients With Double-Seronegative Myasthenia Gravis and Antibodies to Cortactin. JAMA Neurol. 2016;73(9):1099-1104.

21. Luchanok U, Kaminski H. Ocular myasthenia: diagnostic and treatment recommendations and the evidence base. Curr Opin Neurol. 2008;21(1):8-15. 